\title{
Combined immunohistochemical and immunofluorescence method to determine the phenotype of proliferating cell populations
}

\author{
A L Ramshaw, D V Parums
}

\begin{abstract}
Aims: To determine the phenotype of proliferating cell populations.

Methods: The double immunostaining technique combines the autofluorescent properties of alkaline phosphatase substrate naphthol/Fast Red with immunofluorescence using fluorescein. Fresh human tonsil and fresh atherosclerotic aortic aneurysm wall tissue were studied using a panel of monclonal antibodies including Ki-67, CD4, CD8, CD19, CD22, HLA-DRa, CD68 and CD31.

Results: This double immunostaining method permitted simultaneous colocalisation of different markers on the same cell and could be used to identify HLA-DR positive cells as well as proliferation associated Ki-67 positive cells in human tonsil tissue and in chronic periaortitis associated with advanced atherosclerosis.

Conclusion: This technique is simple and the results may be viewed using a single fluorescence filter. The Fast Red reaction product is stable and does not fade under storage. The staining works particularly well with markers for nuclear antigens in combination with markers for cytoplasmic or surface antigens.
\end{abstract}

( Clin Pathol 1992;45:1015-1017)

Both experimental and diagnostic histopathologists have shown increasing interest in the detection of cellular proliferation markers, such as Ki67. ' To determine the phenotype of the proliferating cell populations, double immunohistochemical and double immunofluorescence staining techniques are of great value. Double immunofluorescence in particular can give clear, sensitive results. But two filters need to be used for imaging these results.

In 1960 Burstone described the use of $a$-naphthol substrates in fluorescence techniques for alkaline phosphatase. ${ }^{2}$ As a recent review of published findings has shown, ${ }^{3}$ the use of Fast Red fluorescence, both singly and in combination with other immunofluorescence and immunohistochemical techniques, has been used to solve problems in cellular immunology.

In this paper we describe a simple double immunohistochemical/immunofluorescence technique. This technique makes use of the fact that Fast Red fluoresces red/orange at the same excitation wavelength as fluorescein fluoresces green. It is a double immunostaining technique that uses alkaline phosphatase antialkaline phosphatase (APAAP) and fluorescein isothiocyanate (FITC) conjugated antibodies. The results may be visualised using a single fluorescence filter. This technique is of particular value for showing two different phenotypic markers on the same cell.

We have a particular interest in the chronic inflammatory process which accompanies advanced atherosclerosis, a condition termed "chronic periaortitis". 45 This double staining technique is of value in showing the presence of proliferating lymphocytes in chronic periaortitis.

\section{Methods}

Fresh human tonsil and atherosclerotic abdominal aortic aneurysm wall tissue was obtained at surgery. Frozen tissue was embedded in OCT (Miles Laboratories Inc., Lab-Tek Div., Naperville, Illinois, USA) and then immersed in liquid nitrogen. Cryostat sections 6-9 $\mu \mathrm{m}$ were cut, air dried for 20 hours, and fixed in acetone for 10 minutes. Similar paraffin wax blocks were prepared by tissue fixation in $10 \%$ formalin, followed by routine embedding and sectioning procedures.

Activation and proliferation of cells were observed on serial cryostat and paraffin wax sections using a panel of monoclonal antibodies: Ki-67, HLA-Dra, JC70 (CD31), CD68, CD4, CD8, CD22 and CD19 (table).

Single immunostaining was carried out on serial sections of frozen tissue using the alkaline phosphatase anti-alkaline phosphatase (APAAP) technique. ${ }^{12}$

DOUBLE IMMUNOHISTOCHEMISTRY/IMMUNOFLUORESCENCE

Double immunostaining was carried out to identify HLA-DR positive cells and to identify $\mathrm{Ki}-67$ positive proliferating cells. The monoclonal antibody TAL.1B5 was used in conjunction with monoclonal antibodies to specific cellular markers: macrophage associated antigen (CD68:EBM 11), to the $T$ cytotoxic/suppressor associated phenotype (CD8:Tü102), to the $T$ helper associated phenotype (CD4:T3-10), to the $B$ cell associated phenotype (CD19/22:HD37/ $4 \mathrm{~KB} 128$ ) and to an endothelial cell marker (JC70:CD31). Ki-67 was used in conjunction with monoclonal antibodies to macrophageassociated antigen (CD68:EBM 11), to the T cytotoxic/suppressor associated phenotype
University of Oxford, of Pathology and Radclifie Hy, John Oxford OX3 9DU une 1992 
Panel of monoclonal antibodies

\begin{tabular}{lllc}
\hline Antibody & Specificity & Cell association & Reference \\
\hline Ki-67 & Nuclear antigen & Proliferating cells & 6 \\
TAL.IB5 & HLA-DR $a$ chain & Variety & 7 \\
JC70 & CD31 & Endothelial cells, some platelets, & 8 \\
EBM II & CD68 & Macrophacytes and macrophages & 9 \\
T3-10 & CD4 & T helper cells & 10 \\
Tü102 & CD8 & T cytotoxic cells & 10 \\
HD37 & CD19 & B cells & 11 \\
4KB128 & CD22 & B cells & 11 \\
\hline
\end{tabular}

(CD8:Tü102), to the $T$ helper associated phenotype(CD4:T3-10) and to the $B$ cell associated phenotype (CD19/22:HD37/ 4KB128).

Staining was carried out on serial cryostat sections which were air dried overnight and then fixed in acetone for 10 minutes at room temperature. The first monoclonal antibody (Ki-67, EBM 11, Tü102, T3-10, or HD37/ $4 \mathrm{~KB} 128$ ) was applied as undiluted culture supernatant to dry tissue sections and incubated for one hour. Two subsequent 30 minute incubations were carried out with a 1 in 25 dilution of rabbit anti-mouse immunoglobulin (DAKO) followed by a 1 in 50 dilution of alkaline phosphatase anti-alkaline phosphatase monoclonal antibody (APAAP) (DAKO). The alkaline phosphatase substrate $(0.2 \mathrm{mg} / \mathrm{ml}$ naphthol AS-MX phosphate free acid (Sigma), $0.5 \%$ dimethylformamide, $1 \mathrm{mM}$ levamisole, $1 \mathrm{mg} / \mathrm{ml}$ Fast-Red TR salt (Sigma) in $0.1 \mathrm{M}$ TRIS- $\mathrm{HCl}, \mathrm{pH} 8 \cdot 2$ ) was prepared, filtered, and immediately applied to the sections and was then allowed to develop for 20 minutes.

After washing thoroughly, the sections were incubated in $10 \%$ normal human serum (NHS; heat inactivated and filtered) for 15 minutes followed by the application of the second monoclonal antibody (all combinations with TAL.1B5 or $\mathrm{Ki}-67$ ) for one hour. A 1 in 20 dilution of fluorescein isothiocyanate (FITC) conjugated rabbit anti-mouse immunoglobulin (Dako) in $10 \%$ NHS was then applied and incubated for 30 minutes. Two minute washes in TRIS-buffered saline (TBS; $0.05 \mathrm{M}$ TRIS-HC1 $\mathrm{pH} 7 \cdot 6,0 \cdot 15 \mathrm{M} \mathrm{NaCl}$ ) were carried out between each step. All dilutions were made in TBS and all incubations were carried out at room temperature in a humid chamber.

Sections were mounted in the fluorescein preservative DABCO/glycerol mountant $(90 \%$ glycerol, $10 \%$ TBS, $2 \cdot 3 \%$ 1,4-diazabicyclo(2.2.2)octane (Sigma)) and observed under a Leitz Ortholux II fluorescence microscope with an excitation wavelength between 450 and $490 \mathrm{~nm}$ (fluorescein filter 3). Photographs were taken on Kodak Ektachrome 200 colour reversal film with an exposure of $60-90 \mathrm{sec}-$ onds (figs 1 and 2).

Controls were carried out, using an irrelevant antibody (mouse anti-rabbit immunoglobulin (DAKO)), TBS, NHS and tissue culture media as substitutions at each stage, to exclude the possibility of cross-reaction. Optimal concentrations of secondary antibodies were determined in order to ensure saturating conditions, and single staining, and double stain- ing using every combination of paired antibodies (for example, $\mathrm{Ki}-67$ and EBM II) were carried out.

\section{Results}

Double staining was used to identify and characterise the cell types in the inflamed aortic adventitia and in tonsil. Double staining was also used to determine the phenotype of $\mathrm{Ki}-67$ positive cells in these tissues.

In tonsil tissue, T- and B-lymphocyte populations could be distinguished. Mixed histocompatibility complex (MHC) class II molecule expression was seen in reactive lymphoid tissue and was found by double staining (yellow) to colocalise to B cells, T cells, and occasional endothelial cells. Ki-67 staining was seen in occasional B cells in germinal centres.

In the aortic adventitia, MHC class II expression was observed on various cell types, including on B cells and macrophages, which normally express this antigen, but also on over $50 \%$ of the $\mathrm{T}$ cells (mostly of the T-helper phenotype), on most non-lymphoid smooth muscle-like cells, and on most of the endothelial cells (fig 1).

When lymphoid follicles were present in the aortic adventitia $\mathrm{Ki}-67$ positive $\mathrm{B}$ cells were present, predominantly in a circular pattern around germinal centres, with associated $\mathrm{T}$ cells with the T-helper phenotype (CD4) showing $\mathrm{Ki}-67$ positivity (fig 2 ). No $\mathrm{Ki}-67$ positivity was seen within intimal atheromatous plaques.

\section{Discussion}

A double immunohistochemical/immunofluorescence technique has been described that can be used for the simultaneous detection of different antigens on the same cell. The fluorescence properties of Fast Red have been reported before, ${ }^{23}$ but as far as we know, this technique has not yet been widely used by diagnostic histopathologists. It is a simple method that uses widely available reagents and enhances the usefulness of the alkaline phosphatase technique. The results may be viewed using a single fluorescence filter. The Fast Red reaction product is also stable and does not fade under storage.

By examining the alkaline phosphatase reaction product under bright field and then switching to fluorescence conditions, we have identified $\mathrm{Ki}-67$ positive cells as well as MHC class II positive cells using this method. This technique has been used successfully with monoclonal antibodies of the same species using blocking steps with normal human serum and using optimal antibody concentrations. Staining works particularly well with markers for nuclear antigens in conjunction with markers for cytoplasmic or surface antigens. The use of antibodies from different species would, of course, increase the staining possibilities and virtually eliminate the possibility of cross-reaction. It must be noted, however, that the Fast Red substrate gives a 


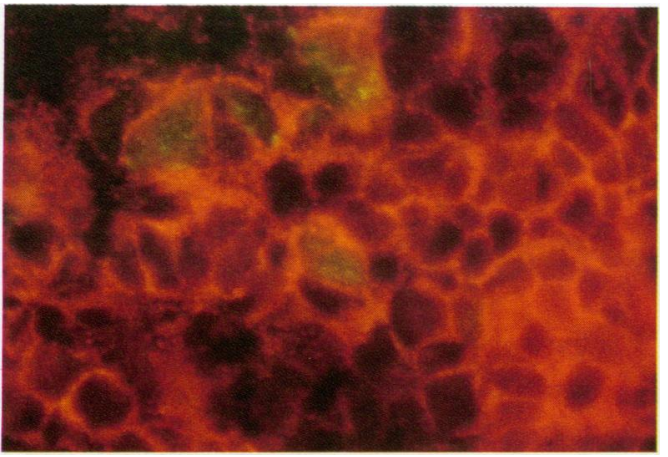

Figure 1 Section of an atherosclerotic abdominal aortic aneurysm showing the chronically inflamed adventitia ('chronic periaortitis'). Double immunostaining has been performed with TAL.1B5 (HLA-DRa) using the APAAP stain (red) and with $\% C 70$ (CD31) with indirect staining using FITC conjugated antibodies (green). In the centre of the figure is a vasa vasorum showing double staining (yellow) of the endothelium which is positive for both CD31 (green) and HLA-DRa (red).

variable colour from red to orange and controls must be used to ensure specificity of the reaction.

This double immunohistochemical and immunofluorescence technique combined with single immunohistochemical staining can demonstrate the presence of proliferating lymphocytes associated with advanced atherosclerosis. The combined immunohistochemical/immunofluorescence method permits the simultaneous detection of different phenotypic markers on the same cell and is particularly useful for the phenotypic identification of cell types associated with nuclear proliferation markers.

We have found this to be a particularly useful method for our work on chronic periaortitis. The findings using these techniques support the view that the inflammation associated with atherosclerosis is-part of an ongoing immune reaction. This may explain why chronic periaortitis has the potential to manifest itself clinically, albeit rarely, as conditions variously termed 'idiopathic retroperitoneal fibrosis' and 'inflammatory aneurysm'.

We thank Professor PJ Morris, Mr P Lamont, and Mr JC Collin of the Nuffield Department of Surgery for providing surgical material; Dr KC Gatter, Dr DY Mason, and Dako A/S for their kind donation of monoclonal antibodies and Professor JO'D

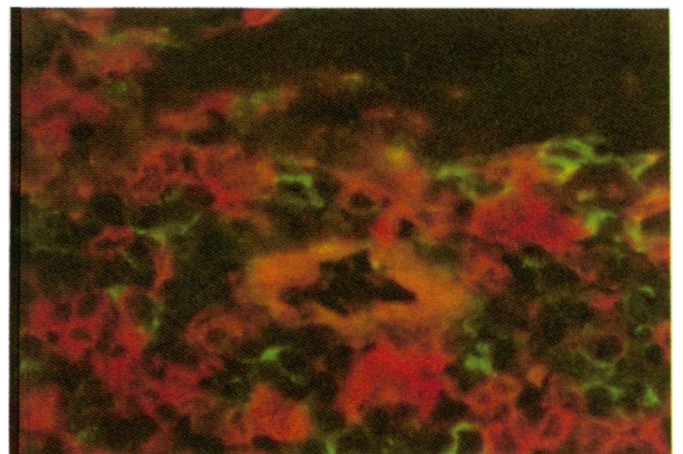

Figure 2 The periphery of a lymphoid follicle within the aortic adventitia from a section of an atherosclerotic aortic aneurysm. Double immunostaining has been performed with T3-10 (CD4) using the APAAP stain (red) and with Ki-67 (proliferation marker) with indirect staining using FITC conjugated antibodies (green). Ki-67 positive (green) staining is present within CD4 positive

(red/orange) cells indicating that cells associated with the T-helper phenotype are proliferating.

McGee for providing laboratory space. This research was funded by the British Heart Foundation.

1 Brown DC, Gatter KC. Monoclonal antibody Ki67: its use in histopathology. Histopathology 1990;17:489-503.

2 Burstone MS. Postcoupling, noncoupling and fluorescence techniques for the demonstration of alkaline phosphatases. fNCI 1960;24:1190-202.

3 Claassen E. Alkaline phosphatase-Fast Red fluorescence. Rediscovery of a useful label? $\mathcal{f}$ Immunol Methods 1991;139:149-50.

4 Parums DV. The spectrum of chronic periaortitis. Histopathology 1990;16:423-31.

5 Ramshaw AL, Parums DV. Immunohistochemical characterization of inflammatory cells associated with advanced atherosclerosis. Histopathology 1990;17:543-52.

6 Gerdes J, Schwab U, Lemke H, Stein H. Production of a mouse monoclonal antibody reactive with a human nuclear antigen associated with cell proliferation. Int 7 Cancer 1983;31:13-20.

7 Epenetos AA, Borrow LG, Adams TE, Collins CM, Isaacson PG, Bodmer WF. A monoclonal antibody that Isaacson PG, Bodmer WF. A monoclonal antibody that detects HLA-D region antigen in routinely fixed, wax tissue. F Clin Pathol 1985;38:12-7.

8 Parums DV, Cordell JL, Micklem K, Heryet AR, Gatter KC, Mason DY. JC70: a new monoclonal antibody that detects vascular endothelium-associated antigen on routinely processed tissue sections. $f$ Clin Pathol 1990;43:752-7.

9 Kelly PMA, Bliss E, Morton JA, Burns J, McGee JO'D. Monoclonal antibody EBM/11: high cellular specificity for human macrophages. $\mathcal{F}$ Clin Pathol 1988;41:510-5.

10 Erber WN, Pinching AJ, Mason DY. Immunocytochemical detection of $T$ and $B$ cell populations in routine blood imears. Lancet 1984;i:1042-5.

11 Stein H, Gerdes J, Mason DY. The normal and malignant germinal centre. Clin Haematol 1982;11:531-9.

12 Cordell JL, Falini B, Erber WN, et al. Immunoenzymatic labelling of monoclonal antibodies using immune complexes of alkaline phosphatase and monoclonal antiplexes of alkaline phosphatase and monoclonal antialkaline phosphatase (APAAP
Cytochem 1984;32:219-22. 


\section{Corrections}

Combined immunohistochemical and immunofluorescence method to determine the phenotype of proliferating cell populations (A L Ramshaw, D V Parums: $f$ Clin Pathol 1992;45:1015-17).

The colour photographs below were inadvertently transposed during the printing process: this is how they should have appeared.

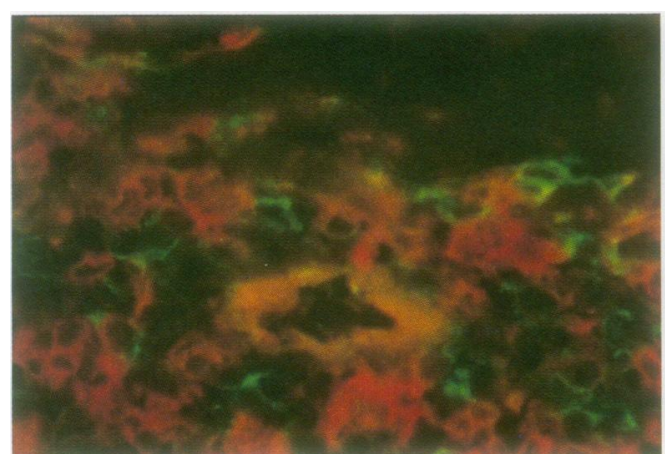

Figure 1 Section of an atherosclerotic abdominal aortic aneurysm showing the chronically inflamed adventitia ('chronic periaortitis'). Double immunostaining has been performed with TAL.1B5 (HLA-DRa) using the APAAP stain (red) and with $\mathcal{F C} 70$ (CD31) with indirect staining using FITC conjugated antibodies (green). In the centre of the figure is a vasa vasorum showing double staining (yellow) of the endothelium which is positive for both $C D 31$ (green) and $H L A-D R a$ (red).

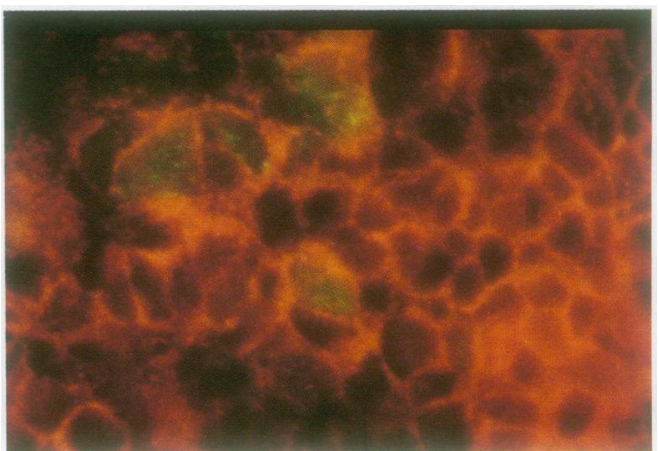

Figure 2 The periphery of a lymphoid follicle within the aortic adventitia from a section of an atherosclerotic aortic aneurysm. Double immunostaining has been performed with T3-10 (CD4) using the APAAP stain (red) and with Ki-67 (proliferation marker) with indirect staining using FITC conjugated antibodies (green). Ki-67 positive (green) staining is present within CD4 positive

(red/orange) cells indicating that cells associated with the T-helper phenotype are proliferating.

A rapid method for the detection of monoclonality in B cell lymphoma in lymph node aspirates using the polymerase chain reaction (P J Sykes, et al: $\mathcal{f}$ Clin Pathol 1992;45:420-3)

A typographical error appeared in the sequence of the FR3A primer in the methods section of the above paper:

It was published as:

FR3A, 5' ACACG G[C/T][G/C] TGTATT ACTGT 3'

It should read:

FR3A, 5' ACACG GC[C/T][G/C] TGTATT ACTGT 3'

NOTICES

\section{Third Congress of Asia Pacific \\ Association of Societies of Pathologists}

14-17 February 1993

At present, the member countries of APASP are Bangladesh, India, Pakistan, Mayanmar, Nepal, New Zealand, Australia and Singapore. Korea, Japan, and Malaysia are the observing countries.

A detailed scientific programme has been chalked out for this congress. There will be three symposia: (a) postgraduate medical education in pathology/laboratory medicine in the developing countries, (b) epidemiology, diagnosis, and prevention of AIDS and (c) cancer control programmes in developing countries. There will be two workshops: the laboratory diagnosis of Kala-azar and on FNAC (fine needle aspiration cytology).

There will be four free paper sessions. Among others, papers on viral hepatitis, tropical diseases including malaria and sexually transmitted diseases apart from AIDS, will be preferred.

Medical professionals from all branches of pathology who wish to participate in this congress are requested to contact directly the secretariat of Third APASP Congress, in the Department of Pathology, Institute of Postgraduate Medicine and Research (PGMR), Dhaka-1000, Bangladesh.

\section{Histopathology for the MRCPath 25-29 January 1993, Oak Hotel, Brighton}

A 5 day intensive residential course for MRCPath candidates will include tuition on general pathology, histopathology, cytopathology, necropsy pathology and other subspecialty areas. The course is organised by the South East Thames Regional Higher Training Committee.

Speakers will include Dr M G Cook, Guildford, Professor PA Hall, St. Thomas's Hospital, Professor N Gibbs, Guildford, Dr S Humphreys, King's College, Dr N Kirkham, Brighton, Professor D Levison, Guy's Hospital, Dr S Lucas, UCHMSM, Dr J Salisbury, King's College.

The course will form the first part of a three year cycle aimed to provide a comprehensive preparation for the MRCPath examination in histopathology.

The registration fee of $£ 375$ includes full accommodation and catering for five days and nights.

For registration, contact: Mrs P Newland, Histopathology Department, Royal Sussex County Hospital, Brighton BN2 5BE. Telephone 0273-696955 extension 4269. Fax 0273-600182.

\section{Call for pathologists}

The South African Institute for Medical Research has vacancies for anatomical pathologists (histopathologists) in its diagnostic service.

The South African Institute for Medical Research is a non-profit and non-government organisation which, in addition to its research and training functions, provides a diagnostic pathology and laboratory medicine service across most of South Africa.

Applicants should be in possession of a medical qualification registrable with the South African Medical and Dental Council, and have a qualification and experience in anatomical pathology.

An ability to communicate effectively in both written and spoken English is essential.

Salary and conditions of service are negotiable, depending on qualifications and experience.

Applications, which should include a curriculum vitae and the names of three referees, should be addressed to Miss Joy Paige, Human Resources Manager, SAIMR, PO Box 1038 , 2000 Johannesburg, South Africa.

Further information about the positions can be obtained from Miss Paige or Dr Doehring at the above address or: telephone $+2711725-0511$, or fax $+2711725-5891$.

\section{ACP Locum Bureau}

The Association of Clinical Pathologists runs a locum bureau for consultant pathologists.

Applicants with the MRCPath who would like to do locums and anyone requiring a locum should contact The General Secretary, 221 Preston Road, Brighton BN1 6SA. Tel: (0273) 561188. Fax: (0273) 541227. 